\title{
Integer powers of anti-bidiagonal Hankel matrices
}

\author{
João Lita da Silva* \\ Department of Mathematics and GeoBioTec \\ Faculty of Sciences and Technology \\ NOVA University of Lisbon \\ Quinta da Torre, 2829-516 Caparica, Portugal
}

\begin{abstract}
In this paper we derive a general expression for integer powers of real upper and lower antibidiagonal matrices with constant anti-diagonals using Chebyshev polynomials. An explicit formula for the inverse of these matrices is also provided.
\end{abstract}

Key words: Hankel matrices, Anti-bidiagonal matrices, Chebyshev polynomials

2010 Mathematics Subject Classification: 15A18, 15B05

\section{Introduction}

The computation of integer powers of square matrices is required in several branches of mathematics such as differential equations, linear dynamical systems, graph theory or numerical analysis (particularly, it is important in some numerical problems in order to know whether a square matrix is convergent or semi-convergent). Recently, some authors studied this subject for a specific type of Hankel matrices (see [5], [6], [7], [8], [11], [12] among others).

The main goal of this paper is to establish an expression for integer powers of real anti-bidiagonal Hankel matrices, i.e. matrices of the form $\operatorname{antitridiag}_{n}(a, c, 0)$ and $\operatorname{antitridiag}_{n}(0, c, b), a, b, c \in \mathbb{R}$, proceeding with the formulas developed in [6] and [7]. Recall that the shape of these matrices, namely the absence of symmetry about the northeast-southwest diagonal in general, makes the approach to this problem be more tricky than the persymmetric case. To overcome this nuisance, we shall employ a useful lemma presented in [6] and suitable results about eigenvalues and eigenvectors of general tridiagonal matrices. This technique will allow us to achieve the desired expressions for integer powers of both upper and lower real anti-bidiagonal Hankel matrices at the expense of Chebyshev polynomials. Additionally, a formula not depending on any unknown parameter for the inverse of complex anti-bidiagonal Hankel matrices will be also presented.

*E-mail address: jfls@fct.unl.pt; joao.lita@gmail.com 


\section{Integer powers: general expression}

We begin this section by reviewing some special types of matrices that play a central role in this paper. We say that an $n \times n$ matrix is upper anti-bidiagonal if it has the form

$$
\left[\begin{array}{ccccccc}
0 & \ldots & \ldots & \ldots & 0 & a_{n} & c_{n} \\
\vdots & & & . \cdot & a_{n-1} & c_{n-1} & 0 \\
\vdots & & . \cdot & . \cdot & c_{n-2} & . \cdot & \vdots \\
\vdots & . \cdot & . \cdot & . \cdot & . \cdot & & \vdots \\
0 & a_{3} & c_{3} & . \cdot & & & \vdots \\
a_{2} & c_{2} & . \cdot & & & & \vdots \\
c_{1} & 0 & \ldots & \ldots & \ldots & \ldots & 0
\end{array}\right]
$$

an $n \times n$ matrix is said to be lower anti-bidiagonal if it has the form

$$
\left[\begin{array}{ccccccc}
0 & \ldots & \ldots & \ldots & \ldots & 0 & c_{n} \\
\vdots & & & & . \cdot & c_{n-1} & b_{n} \\
\vdots & & & . \cdot & c_{n-2} & b_{n-1} & 0 \\
\vdots & & . \cdot & . \cdot & . \cdot & . \cdot & \vdots \\
\vdots & . \cdot & c_{3} & . \cdot & . \cdot & & \vdots \\
0 & c_{2} & b_{3} & . \cdot & & & \vdots \\
c_{1} & b_{2} & 0 & \ldots & \ldots & \ldots & 0
\end{array}\right]
$$

We say that an $n \times n$ matrix is anti-bidiagonal if it is upper anti-bidiagonal or lower anti-bidiagonal. Consider the $n \times n$ upper anti-bidiagonal matrix with constant anti-diagonals

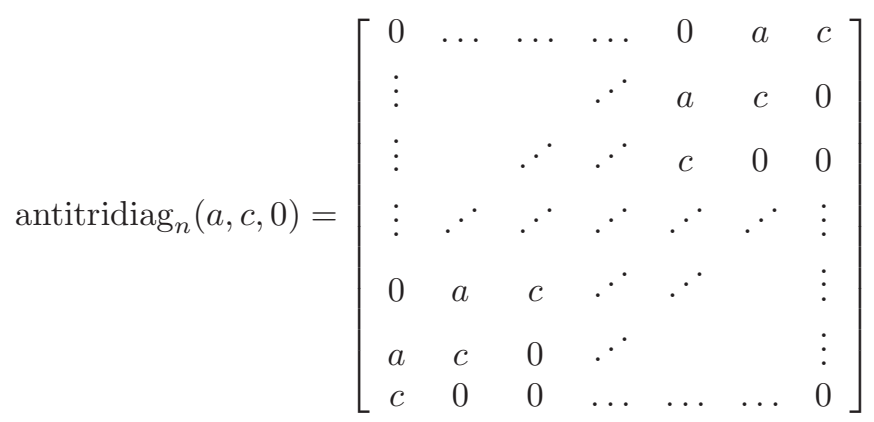

and the following $n \times n$ lower bidiagonal matrix

$$
\operatorname{tridiag}_{n}(a, c, 0)=\left[\begin{array}{ccccccc}
c & 0 & 0 & \ldots & \ldots & \ldots & 0 \\
a & c & 0 & \ddots & & & \vdots \\
0 & a & c & \ddots & \ddots & & \vdots \\
\vdots & \ddots & \ddots & \ddots & \ddots & \ddots & \vdots \\
\vdots & & \ddots & \ddots & c & 0 & 0 \\
\vdots & & & \ddots & a & c & 0 \\
0 & \ldots & \ldots & \ldots & 0 & a & c
\end{array}\right] .
$$


Our first auxiliary result relates integer powers of (2.1) with (2.2) and the $n \times n$ exchange matrix

$$
\mathbf{J}_{n}=\left[\begin{array}{ccccc}
0 & \ldots & \ldots & 0 & 1 \\
\vdots & & . & 1 & 0 \\
\vdots & . \cdot & . \cdot & . \cdot & \vdots \\
0 & 1 & . \cdot & & \vdots \\
1 & 0 & \ldots & \ldots & 0
\end{array}\right]
$$

The proof can be found in [6] (page 1314).

Lemma 1 Let $\mathbf{P}_{n}$ be an $n \times n$ persymmetric matrix ${ }^{\dagger}$ and $\mathbf{S}_{n}=\mathbf{J}_{n} \mathbf{P}_{n}$ where $\mathbf{J}_{n}$ is the $n \times n$ exchange matrix (2.3). If $m$ is a positive integer then

$$
\mathbf{S}_{n}^{m}=\left\{\begin{array}{cl}
\left(\mathbf{P}_{n}^{\top} \mathbf{P}_{n}\right)^{\frac{m}{2}} & \text { if } m \text { is even } \\
\mathbf{J}_{n} \mathbf{P}_{n}\left(\mathbf{P}_{n}^{\top} \mathbf{P}_{n}\right)^{\frac{m-1}{2}} & \text { if } m \text { is odd }
\end{array}\right.
$$

Furthermore, if $\mathbf{P}_{n}$ is nonsingular then

$$
\mathbf{S}_{n}^{-m}=\left\{\begin{array}{cl}
\left(\mathbf{P}_{n}^{\top} \mathbf{P}_{n}\right)^{-\frac{m}{2}} & \text { if } m \text { is even, } \\
\mathbf{J}_{n} \mathbf{P}_{n}\left(\mathbf{P}_{n}^{\top} \mathbf{P}_{n}\right)^{-\frac{m+1}{2}} & \text { if } m \text { is odd }
\end{array}\right.
$$

The next result will permit us to identify the eigenvalues and also eigenvectors of a tridiagonal matrix

$$
\mathbf{M}_{n}=\left[\begin{array}{ccccccc}
c_{1} & b_{1} & 0 & \ldots & \ldots & \ldots & 0 \\
a_{2} & c_{2} & b_{2} & \ddots & & & \vdots \\
0 & a_{3} & c_{3} & \ddots & \ddots & & \vdots \\
\vdots & \ddots & \ddots & \ddots & \ddots & \ddots & \vdots \\
\vdots & & \ddots & \ddots & c_{n-2} & b_{n-2} & 0 \\
\vdots & & & \ddots & a_{n-1} & c_{n-1} & b_{n-1} \\
0 & \ldots & \ldots & \ldots & 0 & a_{n} & c_{n}
\end{array}\right]
$$

where $\left\{a_{k}\right\}_{1 \leqslant k \leqslant n},\left\{b_{k}\right\}_{1 \leqslant k \leqslant n}$ and $\left\{c_{k}\right\}_{1 \leqslant k \leqslant n}$ are sequences of complex numbers such that $b_{k} \neq 0$ and $b_{n}=1$. Supposing the polynomial sequence $\left\{Q_{k}(x)\right\}_{k \geqslant 0}$ characterized by a three-term recurrence relation

$$
\begin{aligned}
& x Q_{0}(x)=c_{1} Q_{0}(x)+b_{1} Q_{1}(x) \\
& x Q_{k-1}(x)=a_{k} Q_{k-2}(x)+c_{k} Q_{k-1}(x)+b_{k} Q_{k}(x) \text { for } k \geqslant 2
\end{aligned}
$$

with initial condition $Q_{0}(x)=1$, we can give a matrix form for this three-term recurrence relation:

$$
x \mathbf{q}(x)=\mathbf{M}_{n} \mathbf{q}(x)+Q_{n}(x) \mathbf{e}
$$

where $\mathbf{M}_{n}$ is given by (2.4),

$$
\mathbf{q}(x)=\left[\begin{array}{c}
Q_{0}(x) \\
Q_{1}(x) \\
\vdots \\
Q_{n-1}(x)
\end{array}\right] \text { and } \mathbf{e}=\left[\begin{array}{c}
0 \\
\vdots \\
0 \\
1
\end{array}\right]
$$

The following statement is a well-known result.

\footnotetext{
${ }^{\dagger}$ An $n \times n$ matrix $\mathbf{P}_{n}$ is said persymmetric if it is symmetric about its northeast-southwest diagonal, i.e. $\left[\mathbf{P}_{n}\right]_{k, \ell}=$ $\left[\mathbf{P}_{n}\right]_{n-\ell+1, n-k+1}$ for all $k, \ell$ (see [2], page 193).
} 
Lemma 2 If $\lambda$ is a zero of the polynomial $Q_{n}(x)$ obtained by (2.5) then $\lambda$ is an eigenvalue of the tridiagonal matrix (2.4) and

$$
\mathbf{q}(\lambda)=\left[\begin{array}{c}
Q_{0}(\lambda) \\
Q_{1}(\lambda) \\
\vdots \\
Q_{n-1}(\lambda)
\end{array}\right]
$$

is a corresponding eigenvector.

Proof. Let $\lambda$ be a zero of the polynomial $Q_{n}(x)$. From (2.6) we have $\mathbf{M}_{n} \mathbf{q}(\lambda)=\lambda \mathbf{q}(\lambda)$ and $\lambda$ is an eigenvalue of $\mathbf{M}_{n}$. Since $Q_{0}(x) \equiv 1 \neq 0$ it follows also that $\mathbf{q}(\lambda)$ is a eigenvector associated to $\lambda$.

Setting $\mathbf{P}_{n}=\operatorname{tridiag}_{n}(a, c, 0)$ with $a c \neq 0$ we have $\mathbf{S}_{n}=\mathbf{J}_{n} \mathbf{P}_{n}=\operatorname{antitridiag}_{n}(a, c, 0)$ and

$$
\mathbf{P}_{n}^{\top} \mathbf{P}_{n}=\left[\begin{array}{cccccccc}
a^{2}+c^{2} & a c & 0 & \cdots & \cdots & \ldots & 0 \\
a c & a^{2}+c^{2} & a c & \ddots & & & \vdots \\
0 & a c & a^{2}+c^{2} & \ddots & & & \vdots \\
\vdots & \ddots & \ddots & \ddots & \ddots & \ddots & \vdots \\
\vdots & & & \ddots & a^{2}+c^{2} & a c & 0 \\
\vdots & & & & \ddots & a c & a^{2}+c^{2} & a c \\
0 & \ldots & \cdots & \cdots & 0 & a c & c^{2}
\end{array}\right]
$$

where $\xi=\frac{a}{c}$. Hence, integer powers of

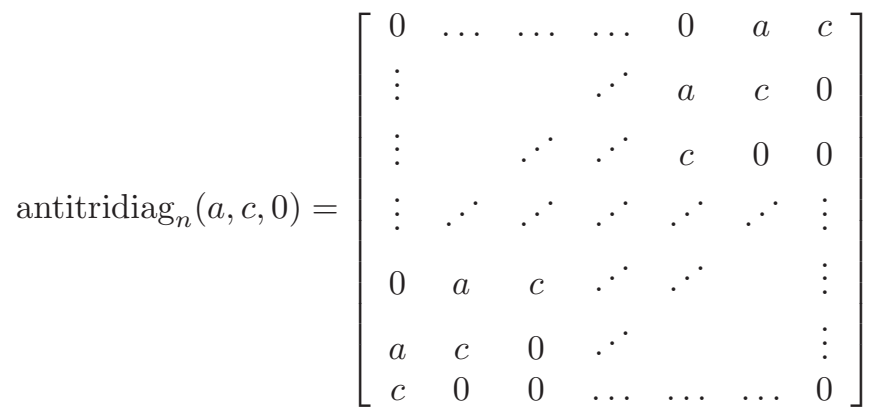


can be computed at the expense of the integer powers of the following tridiagonal $n \times n$ matrix

$$
\mathbf{T}_{n}=\left[\begin{array}{ccccccc}
\xi+\frac{1}{\xi} & 1 & 0 & \ldots & \ldots & \ldots & 0 \\
1 & \xi+\frac{1}{\xi} & 1 & \ddots & & & \vdots \\
0 & 1 & \xi+\frac{1}{\xi} & \ddots & & & \vdots \\
\vdots & \ddots & \ddots & \ddots & \ddots & \ddots & \vdots \\
\vdots & & & \ddots & \xi+\frac{1}{\xi} & 1 & 0 \\
\vdots & & & \ddots & 1 & \xi+\frac{1}{\xi} & 1 \\
0 & \ldots & \ldots & \ldots & 0 & 1 & \frac{1}{\xi}
\end{array}\right] .
$$

Let us observe that the case $c=0$ is already established in [6] (page 1316), as well as the case $a=0$ which was presented in formulae (3a), (3b), (3c) and (3d) of [7].

In [13], the authors established a necessary condition for the eigenvalues of tridiagonal Toeplitz matrices having four corners perturbed giving also the corresponding eigenvectors, and their results can be used on matrix $\mathbf{T}_{n}$. However, our approach involving Chebyshev polynomials takes advantage of many results stated in the scope of the theory of orthogonal polynomials, particularly, numerical methods developed by Grant and Rahman to determine the zeros of a linear combination of Chebyshev polynomials (see [3] and [4], for details).

Denoting by $U_{p}(x), p \geqslant 0$ the $p$ th degree Chebyshev polynomial of the second kind

$$
U_{p}(x)=\frac{\sin [(p+1) \arccos x]}{\sin (\arccos x)}, \quad-1<x<1
$$

with $U_{p}( \pm 1)=( \pm 1)^{p}(p+1)$ (see [9]), the polynomial sequence $\left\{Q_{k}(x)\right\}_{k \geqslant 0}$ defined in (2.5) for the matrix (2.8) will be

$$
Q_{k}(x)=U_{k}\left(\frac{x-\xi-\frac{1}{\xi}}{2}\right), \quad 0 \leqslant k \leqslant n-1
$$

and $Q_{n}(x)=U_{n}\left(\frac{x-\xi-\frac{1}{\xi}}{2}\right)+\xi U_{n-1}\left(\frac{x-\xi-\frac{1}{\xi}}{2}\right)$. It is well-known that the zeros of $Q_{n}(x)$ are all real and simple (see [1], page 27). Thus, setting $\theta_{k}, k=1, \ldots, n$ as the solutions of the equation

$$
U_{n}(t)+\xi U_{n-1}(t)=0,
$$

the result below will provide us important matrices in the computing process of integer powers of real anti-bidiagonal Hankel matrices. As pointed out above, the solutions $\theta_{k}$ can be obtained, at least, using numerical methods (see [3] and [4]).

Lemma 3 Let $n \in \mathbb{N}$ and $a, c \in \mathbb{R} \backslash\{0\}$. If $\left[\mathbf{U}_{n}\right]_{k, \ell}=U_{k-1}\left(\theta_{\ell}\right)$ for all $k, \ell=1, \ldots, n$ with $\theta_{j}$, $j=1, \ldots, n$ the solutions of $(2.9)$ then

$$
\mathbf{U}_{n}^{\top} \mathbf{U}_{n}=\frac{1}{2} \operatorname{diag}\left(\left[U_{n}^{\prime}\left(\theta_{1}\right)+\frac{a}{c} U_{n-1}^{\prime}\left(\theta_{1}\right)\right] U_{n-1}\left(\theta_{1}\right), \ldots,\left[U_{n}^{\prime}\left(\theta_{n}\right)+\frac{a}{c} U_{n-1}^{\prime}\left(\theta_{n}\right)\right] U_{n-1}\left(\theta_{n}\right)\right) .
$$

Proof. Setting $\xi=\frac{a}{c}$ we have

$$
\left[\mathbf{U}_{n}^{\top} \mathbf{U}_{n}\right]_{k, \ell}=\sum_{j=0}^{n-1} Q_{j}\left(\xi+\frac{1}{\xi}+2 \theta_{k}\right) Q_{j}\left(\xi+\frac{1}{\xi}+2 \theta_{\ell}\right)=\sum_{j=0}^{n-1} U_{j}\left(\theta_{k}\right) U_{j}\left(\theta_{\ell}\right)
$$

where $\theta_{k}, k=1, \ldots, n$ are the solutions of (2.9). From Christoffel-Darboux identities (see [1], pages 23 and 24 ) we obtain

$$
\sum_{j=0}^{n-1} Q_{j}\left(\xi+\frac{1}{\xi}+2 \theta_{k}\right) Q_{j}\left(\xi+\frac{1}{\xi}+2 \theta_{\ell}\right)=\frac{Q_{n}\left(\xi+\frac{1}{\xi}+2 \theta_{k}\right) Q_{n-1}\left(\xi+\frac{1}{\xi}+2 \theta_{\ell}\right)-Q_{n-1}\left(\xi+\frac{1}{\xi}+2 \theta_{k}\right) Q_{n}\left(\xi+\frac{1}{\xi}+2 \theta_{\ell}\right)}{2\left(\theta_{k}-\theta_{\ell}\right)}
$$




$$
\begin{aligned}
& =\frac{\left[U_{n}\left(\theta_{k}\right)+\xi U_{n-1}\left(\theta_{k}\right)\right] U_{n-1}\left(\theta_{\ell}\right)-U_{n-1}\left(\theta_{k}\right)\left[U_{n}\left(\theta_{\ell}\right)+\xi U_{n-1}\left(\theta_{\ell}\right)\right]}{2\left(\theta_{k}-\theta_{\ell}\right)} \\
& =0
\end{aligned}
$$

for each $k, \ell=1, \ldots, n$ such that $k \neq \ell$ since from (2.9) we have $U_{n}\left(\theta_{k}\right)+\xi U_{n-1}\left(\theta_{k}\right)=0$ for all $k=1, \ldots, n$. On the other hand, using the confluent form of the Christoffel-Darboux identity,

$$
\begin{aligned}
\sum_{j=0}^{n-1} Q_{j}^{2}\left(\xi+\frac{1}{\xi}+2 \theta_{k}\right) & =\frac{1}{2}\left[U_{n}^{\prime}\left(\theta_{k}\right)+\xi U_{n-1}^{\prime}\left(\theta_{k}\right)\right] U_{n-1}\left(\theta_{k}\right)-\frac{1}{2} U_{n}\left(\theta_{k}\right) U_{n-1}^{\prime}\left(\theta_{k}\right) \\
& =\frac{1}{2}\left[U_{n}^{\prime}\left(\theta_{k}\right)+\xi U_{n-1}^{\prime}\left(\theta_{k}\right)\right] U_{n-1}\left(\theta_{k}\right)
\end{aligned}
$$

for each $k=1, \ldots, n$, which establishes the thesis.

Remark The values involving $U_{p}^{\prime}(x)$ can be computed using the Chebyshev polynomials of the second kind since

$$
U_{p}^{\prime}(x)=\left\{\begin{array}{cc}
\frac{(p+2) U_{p-1}(x)-p U_{p+1}(x)}{2\left(1-x^{2}\right)} & \text { if } p>0 \\
0 & \text { if } p=0
\end{array}\right.
$$

and $U_{p}^{\prime}( \pm 1)=( \pm 1)^{p+1} \frac{(p+1)^{3}-(p+1)}{3}$ whenever $p>0$. Moreover, the matrix $\mathbf{U}_{n}$ of Lemma 3 is nonsingular since $\left[U_{n}^{\prime}\left(\theta_{k}\right)+\frac{a}{c} U_{n-1}^{\prime}\left(\theta_{k}\right)\right] U_{n-1}\left(\theta_{k}\right)>0$ for all $k=1, \ldots, n$ (see [1], page 24), yielding

$$
\mathbf{U}_{n}^{-1}=\operatorname{diag}\left(\frac{2}{\left[U_{n}^{\prime}\left(\theta_{1}\right)+\frac{a}{c} U_{n-1}^{\prime}\left(\theta_{1}\right)\right] U_{n-1}\left(\theta_{1}\right)}, \ldots, \frac{2}{\left[U_{n}^{\prime}\left(\theta_{n}\right)+\frac{a}{c} U_{n-1}^{\prime}\left(\theta_{n}\right)\right] U_{n-1}\left(\theta_{n}\right)}\right) \mathbf{U}_{n}^{\top} .
$$

The result below will give us positive integer powers of real anti-bidiagonal Hankel matrices.

Theorem 1 Let $a, c \in \mathbb{R} \backslash\{0\}$ and $n, m \in \mathbb{N}$. If $\mathbf{U}_{n}=\left[U_{k-1}\left(\theta_{\ell}\right)\right]_{k, \ell}, k, \ell=1, \ldots, n$ and $\boldsymbol{\Theta}_{n}=$ $\operatorname{diag}\left(\frac{a}{c}+\frac{c}{a}+2 \theta_{1}, \ldots, \frac{a}{c}+\frac{c}{a}+2 \theta_{n}\right)$ with $\theta_{j}, j=1, \ldots, n$ the solutions of $(2.9)$ then $\mathbf{U}_{n} \boldsymbol{\Theta}_{n} \mathbf{U}_{n}^{-1}$ is an eigenvalue decomposition of the $n \times n$ matrix $\mathbf{T}_{n}$ in (2.8). Moreover, if $\mathbf{A}_{n}:=\operatorname{antitridiag}_{n}(a, c, 0)$ then

$$
\mathbf{A}_{n}^{m}=\left\{\begin{array}{l}
(a c)^{\frac{m}{2}} \mathbf{U}_{n} \boldsymbol{\Theta}_{n}^{\frac{m}{2}} \mathbf{D}_{n} \mathbf{U}_{n}^{\top} \text { if } m \text { is even, } \\
(a c)^{\frac{m-1}{2}} \mathbf{A}_{n} \mathbf{U}_{n} \boldsymbol{\Theta}_{n}^{\frac{m-1}{2}} \mathbf{D}_{n} \mathbf{U}_{n}^{\top} \text { if } m \text { is odd }
\end{array}\right.
$$

where $\mathbf{D}_{n}=\operatorname{diag}\left(\frac{2}{\left[U_{n}^{\prime}\left(\theta_{1}\right)+\frac{a}{c} U_{n-1}^{\prime}\left(\theta_{1}\right)\right] U_{n-1}\left(\theta_{1}\right)}, \ldots, \frac{2}{\left[U_{n}^{\prime}\left(\theta_{n}\right)+\frac{a}{c} U_{n-1}^{\prime}\left(\theta_{n}\right)\right] U_{n-1}\left(\theta_{n}\right)}\right)$.

Proof. Let $\xi=\frac{a}{c}$ and $\theta_{1}, \theta_{2}, \ldots, \theta_{n}$ the solutions of (2.9). From Lemma 2 each $\xi+\frac{1}{\xi}+2 \theta_{k}, k=1, \ldots, n$ is an eigenvalue of $\mathbf{T}_{n}$ and

$$
\mathbf{u}_{k}=\left[\begin{array}{c}
Q_{0}\left(\xi+\frac{1}{\xi}+2 \theta_{k}\right) \\
Q_{1}\left(\xi+\frac{1}{\xi}+2 \theta_{k}\right) \\
\vdots \\
Q_{n-1}\left(\xi+\frac{1}{\xi}+2 \theta_{k}\right)
\end{array}\right]=\left[\begin{array}{c}
U_{0}\left(\theta_{k}\right) \\
U_{1}\left(\theta_{k}\right) \\
\vdots \\
U_{n-1}\left(\theta_{k}\right)
\end{array}\right]
$$

is a corresponding (non-null) eigenvector of $\mathbf{T}_{n}$ associated to $\xi+\frac{1}{\xi}+2 \theta_{k}$. Hence, $\left\{\mathbf{u}_{1}, \mathbf{u}_{2}, \ldots, \mathbf{u}_{n}\right\}$ is a complete set of eigenvectors of $\mathbf{T}_{n}$ (see [10], page 507) leading to $\mathbf{U}_{n}=\left[\begin{array}{lllll}\mathbf{u}_{1} & \mathbf{u}_{2} & \ldots & \mathbf{u}_{n}\end{array}\right]$ and $\mathbf{T}_{n}=\mathbf{U}_{n} \boldsymbol{\Theta}_{n} \mathbf{U}_{n}^{-1}$. The last sentence follows directly from Lemma 3 (and subsequent Remark).

Remark The formulas announced in Theorem 1 can be extended to any negative integer $m$ since

$$
\operatorname{det}\left(\mathbf{A}_{n}\right)=\operatorname{det}\left(\mathbf{J}_{n}\right) \cdot \operatorname{det}\left[\operatorname{tridiag}_{n}(a, c, 0)\right]=c^{n}(-1)^{\frac{n(n-1)}{2}} \neq 0 .
$$


From Theorem 1, we can now compute the entries of any integer power of a real upper antibidiagonal Hankel matrix using the solutions of (2.9) and Chebyshev polynomials of the second kind. The main result of this paper follows next.

Theorem 2 Let $a, c \in \mathbb{R} \backslash\{0\}$ and $n, m \in \mathbb{N}$. If $\mathbf{A}_{n}:=\operatorname{antitridiag}_{n}(a, c, 0)$ and $\theta_{j}, j=1, \ldots, n$ are the solutions of $(2.9)$ then

$$
\left[\mathbf{A}_{n}^{ \pm m}\right]_{k, \ell}=2(a c)^{ \pm \frac{m}{2}} \sum_{j=1}^{n} \frac{\left(\frac{a}{c}+\frac{c}{a}+2 \theta_{j}\right)^{ \pm \frac{m}{2}}}{\left[U_{n}^{\prime}\left(\theta_{j}\right)+\frac{a}{c} U_{n-1}^{\prime}\left(\theta_{j}\right)\right] U_{n-1}\left(\theta_{j}\right)} U_{k-1}\left(\theta_{j}\right) U_{\ell-1}\left(\theta_{j}\right)
$$

when $m$ is even and

$$
\left[\mathbf{A}_{n}^{ \pm m}\right]_{k, \ell}=\left\{\begin{array}{l}
2(a c)^{\frac{ \pm m-1}{2}} \sum_{j=1}^{n} \frac{\left(\frac{a}{c}+\frac{c}{a}+2 \theta_{j}\right)^{\frac{ \pm m-1}{2}}}{\left[U_{n}^{\prime}\left(\theta_{j}\right)+\frac{a}{c} U_{n-1}^{\prime}\left(\theta_{j}\right)\right] U_{n-1}\left(\theta_{j}\right)}\left[a U_{n-k-1}\left(\theta_{j}\right)+c U_{n-k}\left(\theta_{j}\right)\right] U_{\ell-1}\left(\theta_{j}\right) \text { if } k<n \\
2 c(a c)^{\frac{ \pm m-1}{2}} \sum_{j=1}^{n} \frac{\left(\frac{a}{c}+\frac{c}{a}+2 \theta_{j}\right)^{\frac{ \pm m-1}{2}}}{\left[U_{n}^{\prime}\left(\theta_{j}\right)+\frac{a}{c} U_{n-1}^{\prime}\left(\theta_{j}\right)\right] U_{n-1}\left(\theta_{j}\right)} U_{\ell-1}\left(\theta_{j}\right) \text { if } k=n
\end{array}\right.
$$

whenever $m$ is odd for all $k, \ell=1, \ldots, n$.

With the aid of Sherman-Morrison formula, we shall be able to give an explicit expression for the inverse of $\operatorname{antitridiag}_{n}(a, c, 0)$.

Theorem 3 Let $n \in \mathbb{N}$ and $a, c \in \mathbb{C} \backslash\{0\}$. If $\mathbf{A}_{n}=\operatorname{antitridiag}_{n}(a, c, 0), \frac{a}{c}+\frac{c}{a}+2 \cos \left(\frac{k \pi}{n+1}\right) \neq 0$ for all $k=1, \ldots, n$ and $\frac{2}{n+1} \sum_{k=1}^{n} \frac{\sin ^{2}\left(\frac{k \pi}{n+1}\right)}{\frac{a}{c}+\frac{c}{a}+2 \cos \left(\frac{k \pi}{n+1}\right)} \neq \frac{c}{a}$ then

$$
\mathbf{A}_{n}^{-1}=(a c)^{-1} \mathbf{A}_{n} \mathbf{V}_{n} \mathbf{B}_{n} \mathbf{V}_{n}
$$

where $\left[\mathbf{V}_{n}\right]_{k, \ell}:=\sqrt{\frac{2}{n+1}} \sin \left(\frac{k \ell \pi}{n+1}\right)$ and

$$
\left[\mathbf{B}_{n}\right]_{k, \ell}:=\frac{\delta_{k, \ell}}{\frac{a}{c}+\frac{c}{a}+2 \cos \left(\frac{k \pi}{n+1}\right)}+\frac{2 a(-1)^{k+\ell} \sin \left(\frac{k \pi}{n+1}\right) \sin \left(\frac{\ell \pi}{n+1}\right)}{(n+1)\left[\frac{a}{c}+\frac{c}{a}+2 \cos \left(\frac{k \pi}{n+1}\right)\right]\left[\frac{a}{c}+\frac{c}{a}+2 \cos \left(\frac{\ell \pi}{n+1}\right)\right]\left[c-\frac{2 a}{n+1} \sum_{j=1}^{n} \frac{\sin ^{2}\left(\frac{j \pi}{n+1}\right)}{\frac{a}{a}+2 \cos \left(\frac{j \pi}{n+1}\right)}\right]}
$$

with $\delta_{k, \ell}$ the Kronecker delta.

Proof. Suppose $n \in \mathbb{N}$ and $a, c \in \mathbb{C} \backslash\{0\}$. Setting

$$
\begin{gathered}
\boldsymbol{\Delta}_{n}:=\operatorname{diag}\left[\frac{a}{c}+\frac{c}{a}+2 \cos \left(\frac{\pi}{n+1}\right), \ldots, \frac{a}{c}+\frac{c}{a}+2 \cos \left(\frac{n \pi}{n+1}\right)\right] \\
\widehat{\mathbf{T}}_{n}:=\left[\begin{array}{ccccccc}
\frac{a}{c}+\frac{c}{a} & 1 & 0 & \ldots & \ldots & \ldots & 0 \\
1 & \frac{a}{c}+\frac{c}{a} & 1 & \ddots & & & \vdots \\
0 & 1 & \frac{a}{c}+\frac{c}{a} & \ddots & & & \vdots \\
\vdots & \ddots & \ddots & \ddots & \ddots & \ddots & \vdots \\
\vdots & & & \ddots & \frac{a}{c}+\frac{c}{a} & 1 & 0 \\
\vdots & & & \ddots & 1 & \frac{a}{c}+\frac{c}{a} & 1 \\
0 & \ldots & \ldots & \ldots & 0 & 1 & \frac{a}{c}+\frac{c}{a}
\end{array}\right]
\end{gathered}
$$


and

$$
\widehat{\mathbf{E}}_{n}:=\left[\begin{array}{ccccc}
0 & 0 & \ldots & \ldots & 0 \\
0 & 0 & \ddots & & \vdots \\
\vdots & \ddots & \ddots & \ddots & \vdots \\
\vdots & & \ddots & 0 & 0 \\
0 & \ldots & \ldots & 0 & -\frac{a}{c}
\end{array}\right]
$$

we obtain $\mathbf{V}_{n} \mathbf{T}_{n} \mathbf{V}_{n}=\mathbf{V}_{n}\left(\widehat{\mathbf{T}}_{n}+\widehat{\mathbf{E}}_{n}\right) \mathbf{V}_{n}=\boldsymbol{\Delta}_{n}-\frac{a}{c} \mathbf{w} \mathbf{w}^{\top}$ where

$$
\mathbf{w}=\left[\begin{array}{c}
-\sqrt{\frac{2}{n+1}} \sin \left(\frac{\pi}{n+1}\right) \\
\sqrt{\frac{2}{n+1}} \sin \left(\frac{2 \pi}{n+1}\right) \\
\vdots \\
(-1)^{n} \sqrt{\frac{2}{n+1}} \sin \left(\frac{n \pi}{n+1}\right)
\end{array}\right]
$$

and $\mathbf{T}_{n}$ is the matrix (2.8). Since $\mathbf{V}_{n}$ satisfies $\mathbf{V}_{n}^{2}=\mathbf{I}_{n}$ and $\mathbf{V}_{n}=\mathbf{V}_{n}^{-1}$, it follows

$$
\mathbf{T}_{n}^{-1}=\mathbf{V}_{n}\left[\boldsymbol{\Delta}_{n}^{-1}+\frac{a}{c}\left(1-\frac{a}{c} \mathbf{w}^{\top} \boldsymbol{\Delta}_{n}^{-1} \mathbf{w}\right)^{-1} \boldsymbol{\Delta}_{n}^{-1} \mathbf{w} \mathbf{w}^{\top} \boldsymbol{\Delta}_{n}^{-1}\right] \mathbf{V}_{n}
$$

according to the Sherman-Morrison formula (see [10], page 124). Thus, Lemma 1 implies

$$
\mathbf{A}_{n}^{-1}=\mathbf{J}_{n} \operatorname{tridiag}_{n}(a, c, 0) \mathbf{V}_{n}\left\{(a c)^{-1}\left[\boldsymbol{\Delta}_{n}^{-1}+\frac{a}{c}\left(1-\frac{a}{c} \mathbf{w}^{\top} \boldsymbol{\Delta}_{n}^{-1} \mathbf{w}\right)^{-1} \boldsymbol{\Delta}_{n}^{-1} \mathbf{w} \mathbf{w}^{\top} \boldsymbol{\Delta}_{n}^{-1}\right]\right\} \mathbf{V}_{n}
$$

where $\mathbf{J}_{n}$ is the exchange matrix (2.3), and (2.12) is established. The proof is complete.

Remark Note that the decomposition $\mathbf{T}_{n}=\mathbf{V}_{n}\left(\boldsymbol{\Delta}_{n}-\frac{a}{c} \mathbf{w} \mathbf{w}^{\top}\right) \mathbf{V}_{n}$ leads to

$$
\mathbf{A}_{n}^{m}=\left\{\begin{array}{cl}
(a c)^{\frac{m}{2}} \mathbf{V}_{n}\left(\boldsymbol{\Delta}_{n}-\frac{a}{c} \mathbf{w} \mathbf{w}^{\top}\right)^{\frac{m}{2}} \mathbf{V}_{n} & \text { if } m \text { is even, } \\
(a c)^{\frac{m-1}{2}} \operatorname{antitridiag}_{n}(a, c, 0) \mathbf{V}_{n}\left(\boldsymbol{\Delta}_{n}-\frac{a}{c} \mathbf{w} \mathbf{w}^{\top}\right)^{\frac{m-1}{2}} \mathbf{V}_{n} & \text { if } m \text { is odd }
\end{array}\right.
$$

and

$$
\mathbf{A}_{n}^{-m}=\left\{\begin{array}{cl}
(a c)^{-\frac{m}{2}} \mathbf{V}_{n}\left(\boldsymbol{\Delta}_{n}-\frac{a}{c} \mathbf{w} \mathbf{w}^{\top}\right)^{-\frac{m}{2}} \mathbf{V}_{n} & \text { if } m \text { is even, } \\
(a c)^{-\frac{m+1}{2}} \operatorname{antitridiag}_{n}(a, c, 0) \mathbf{V}_{n}\left(\boldsymbol{\Delta}_{n}-\frac{a}{c} \mathbf{w} \mathbf{w}^{\top}\right)^{-\frac{m+1}{2}} \mathbf{V}_{n} & \text { if } m \text { is odd }
\end{array}\right.
$$

for any positive integer $m$. Furthermore, it is possible to express the eigenvalues of $\boldsymbol{\Delta}_{n}-\frac{a}{c} \mathbf{w} \mathbf{w}^{\top}$ as the zeros of explicit rational functions and the corresponding eigenvectors, which allows us to perform an approach similar to the one presented in [8]. Thereby, an alternative procedure to compute the integer powers of $\mathbf{A}_{n}=\operatorname{antitridiag}_{n}(a, c, 0)$ is also founded.

The formulae established throughout for integer powers of real upper anti-bidiagonal Hankel matrices can be used to compute also integer powers of real lower anti-bidiagonal Hankel matrices,

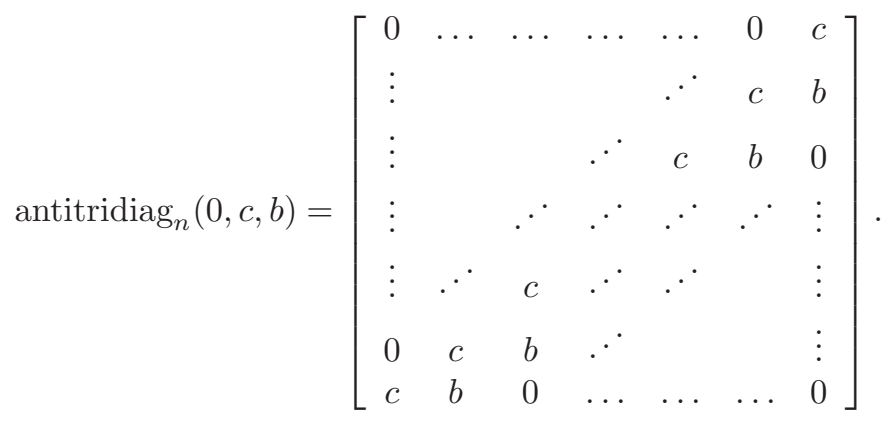


Indeed,

$$
\operatorname{antitridiag}_{n}(0, c, b)=\mathbf{J}_{n} \text { antitridiag }_{n}(b, c, 0) \mathbf{J}_{n},
$$

so that, for any integer $m$ we have $\left[\operatorname{antitridiag}_{n}(0, c, b)\right]^{m}=\mathbf{J}_{n}\left[\operatorname{antitridiag}_{n}(b, c, 0)\right]^{m} \mathbf{J}_{n}$, i.e.

$$
\left\{\left[\operatorname{antitridiag}_{n}(0, c, b)\right]^{m}\right\}_{k, \ell}=\left\{\left[\operatorname{antitridiag}_{n}(b, c, 0)\right]^{m}\right\}_{n-k+1, n-\ell+1}
$$

and similar formulas to $(2.10),(2.11)$ and $(2.12)$ can be obtained for lower anti-bidiagonal Hankel matrices.

Acknowledgements. This work is a contribution to the Project UID/GEO/04035/2013, funded by FCT - Fundação para a Ciência e a Tecnologia, Portugal.

\section{References}

[1] T. S. Chihara, An Introduction to Orthogonal Polynomials, Gordon and Breach, New York (1978).

[2] G. H. Golub and C. F. Van Loan, Matrix Computations, The Johns Hopkins University Press, Baltimore (1996).

[3] J. A. Grant and A. A. Rahman, Determination of the zeros of a linear combination of Chebyshev polynomials, IMA J. Numer. Anal., 3 (1983), 193-206.

[4] J. A. Grant and A. A. Rahman, Determination of the zeros of a linear combination of generalised polynomials, J. Comput. Appl. Math. 42 (1992), 269-278.

[5] J. Gutiérrez-Gutiérrez, Powers of complex persymmetric or skew-persymmetric anti-tridiagonal matrices with constant anti-diagonals, Appl. Math. Comput. 217 (2011), 6125-6132.

[6] J. Lita da Silva, Integer powers of anti-tridiagonal matrices of the form $\operatorname{antitridiag}_{n}(a, 0, b)$, $a, b \in \mathbb{R}$, Comput. Math. Appl. 69 (2015), 1313-1328.

[7] J. Lita da Silva, Integer powers of anti-tridiagonal matrices of the form $\operatorname{antitridiag}_{n}(a, c,-a)$, $a, c \in \mathbb{C}$, Int. J. Comput. Math. 93(10) (2015), 1723-1740.

[8] J. Lita da Silva, On anti-pentadiagonal persymmetric Hankel matrices with perturbed corners, Comput. Math. Appl. 72 (2016), 415-426.

[9] J. C. Mason and D. Handscomb, Chebyshev Polynomials, Chapman \& Hall/CRC, Boca Raton (2003).

[10] C.D. Meyer, Matrix Analysis Applied Linear Algebra, Society for Industrial and Applied Mathematics, Philadelphia (2000).

[11] J. Rimas, Integer powers of real odd order skew-persymmetric anti-tridiagonal matrices with constant anti-diagonals $\left(\operatorname{antitridiag}_{n}(a, c,-a), a \in R \backslash\{0\}, c \in R\right.$ ), Appl. Math. Comput. 219 (2013), 7075-7088.

[12] J. Rimas, Integer powers of real even order anti-tridiagonal Hankel matrices of the form $\operatorname{antitridiag~}_{n}(a, c,-a)$, Appl. Math. Comput. 225 (2013), 204-215.

[13] W. C. Yueh and S. S. Cheng, Explicit eigenvalues and inverses of tridiagonal Toeplitz matrices with four perturbed corners, ANZIAM J. 49 (2008), 361-387. 